\title{
CASE STUDY ON COMBINED LIDAR-PHOTOMETER RETRIEVAL OF VOLCANIC ASH PROPERTIES
}

\author{
Josef Gasteiger $^{1 *}$, Matthias Wiegner ${ }^{1}$, Carlos Toledano ${ }^{2}$, Silke Groß $^{3}$, Volker Freudenthaler ${ }^{1}$ \\ ${ }^{1}$ Meteorologisches Institut, Ludwig-Maximilians-Universität, 80333 München, Germany \\ *Email: josef.gasteiger@lmu.de \\ ${ }^{2}$ Grupo de Óptica Atmosférica, Universidad de Valladolid, Valladolid, Spain \\ ${ }^{3}$ Institut für Physik der Atmosphäre, Deutsches Zentrum für Luft- und Raumfahrt, Oberpfaffenhofen,
} Germany

\begin{abstract}
We study the benefit of considering sun-/skyphotometer measurements in a microphysical lidar retrieval. Furthermore, to assess the importance of the aerosol model employed by the retrieval, we compare results obtained using a spheroid aerosol model with results using an advanced aerosol model that considers irregular particle shapes. Preliminary results are shown for the massextinction conversion factor and the single scattering albedo during a measurement case of long-range-transported volcanic ash.
\end{abstract}

\section{INTRODUCTION}

It had been shown that multi-wavelengths Ramanand depolarization-lidar-systems can provide aerosol data that is sufficient for the retrieval of microphysical properties of volcanic ash [1]. The uncertainties of such retrievals are quite large because of limitations of the information content of the lidar data and also because of assumptions required in the aerosol model employed by the retrieval. Combining vertically-resolving lidar measurements with coincident ground-based sun/sky-photometer measurements seems to be a promising approach to reduce the retrieval uncertainties because of increased information content.

Lopatin et al. [2] developed a combined lidarphotometer retrieval based on the AERONET photometer algorithm extended by consideration of multi-wavelength backscatter coefficients from lidar. They found that the lidar ratio can be retrieved with improved accuracy if the lidar data is considered in addition to the photometer data.

In the present study, we compare retrieval uncertainties of a lidar-only retrieval with uncertainties when photometer data is considered in addition by the retrieval. Furthermore, we compare the retrieval results when a spheroid aerosol model is used with results when a more complex model is used. For these comparisons, we consider the mass-extinction conversion factor $\eta=M / \alpha_{\text {ext }}$, which allows one to calculate the particle mass concentration $M$ from extinction coefficients $\alpha_{\text {ext }}$ (e.g. provided by advanced lidar systems), and the single scattering albedo, which is relevant for radiative transfer. We use measurement data of long-range transported volcanic ash at Maisach/Munich (Germany) in April 2010 for this study.

\section{METHODOLOGY}

Our retrieval approach is based on the lidar retrieval scheme described by [1] and is extended by an improved model for aerosol ensembles and consideration of photometer measurements. It uses the Monte-Carlo method for sampling the microphysical parameters of aerosol ensembles. By comparing modeled results with measurement data, solutions of the retrieval are found.

In the present study we consider two aerosol models. The first model (ash model A) describes particle ensembles consisting of prolate and oblate spheroids. The particle size distributions are mono-modal log-normal distributions. The refractive index is wavelength-independent and does not vary within an ensemble. The particle aspect ratio distributions are described by modified log-normal distributions. Model A is described in detail in Section 3 of [1].

The second model (ash model B) describes ensembles of irregularly-shaped particles, including deformed spheroids, aggregates, and edged particles as shown in Fig. 1 of [3]. The optical properties of these particles were calculated using the Discrete Dipole Approximation [4] up to a size parameter $x$ (= 
$2 \pi r / \lambda)$ of about 20 . For larger particles $(x>20)$, it is assumed that the lidar ratio and linear depolarization ratio is the same as for $x=20$. For the sky radiance simulations required for the photometer retrieval part, large irregularly-shaped particles $(x>20)$ are substituted by prolate spheroids with a prescribed wide aspect ratio distribution (with median around 2.0 and maximum 5.0). Model B ensembles consist of two independent modes. The size distribution of each mode is a log-normal distribution. The refractive index is wavelength-dependent as given by the refractive indices of the mineral components of OPAC [5]. To account for the natural variability, the real part of the refractive index is allowed to vary by \pm 0.04 from OPAC mineral, and the imaginary part is multiplied with a random number in the range from 0 to 1 . Furthermore, absorbing and non-absorbing particles can be mixed within each mode with the mode-average imaginary part not changed and the relative number of non-absorbing particles in the range from 0 to 0.5 .

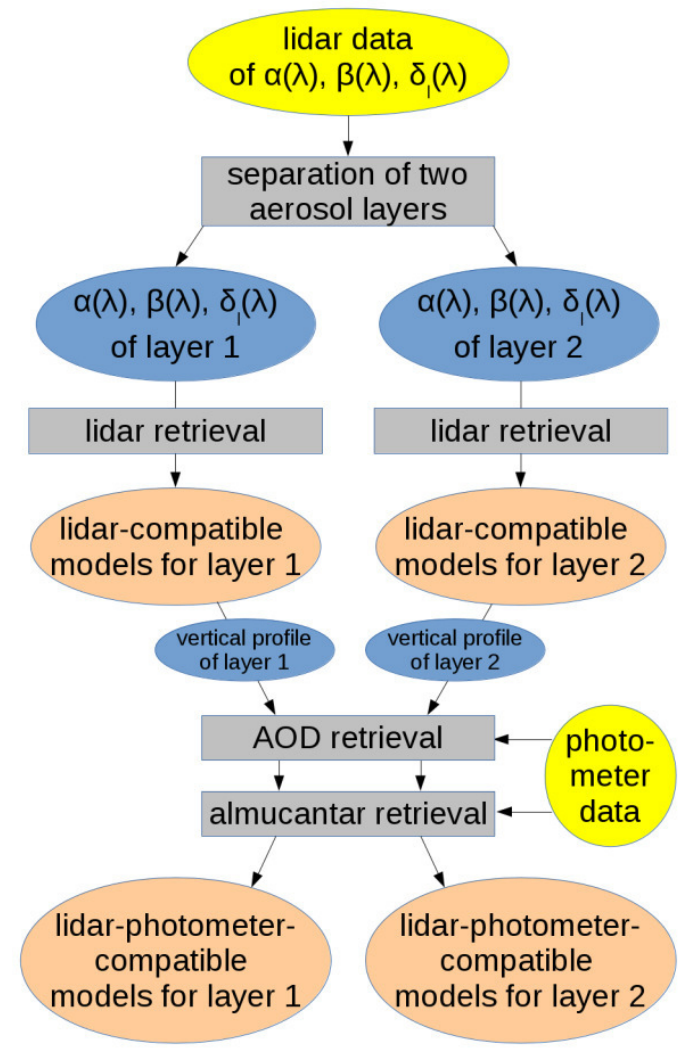

Fig. 1: Simplified flow chart for lidar-photometer retrieval in case of two aerosol layers.
A simplified flow chart of the combined lidarphotometer-retrieval is shown in Fig. 1. As the first step, the lidar profile can be separated into layers based on the intensive aerosol properties. Each aerosol layer is then determined by its (height-independent) intensive optical properties and its vertical structure. For each aerosol layer, the lidar retrieval is applied independently. The aerosol models that are compatible with the lidar measurements are stored for each layer. As the next step, the lidar-compatible ensembles of each layer are combined to atmospheric setups using the extinction coefficient of each layer at a reference wavelength. The wavelength dependence of the extinction is modeled for each ensemble to calculate the wavelength-dependent aerosol optical thickness (AOD) of each atmospheric setup. The AODs are compared with the spectral AOD measured by the photometer. For the setups that agree with the AOD measurements within the measurement uncertainties, radiative transfer calculations are started. The DISORT solver with intensity correction [6], which is included in the libRadtran toolbox [7], is used to model sky radiances in the almucantar geometry at the angles and wavelengths of the photometer. The ground albedo is varied between 0.0 and 0.4. The simulated and measured sky radiances are compared and the setups that agree within the measurement uncertainties are accepted as solutions of the combined lidar-photometer retrieval. As a result, the AOD and the sky radiance measurements are used for refining the set of solutions from the lidar part.

For the present study measurement uncertainties of 5\% for the aerosol optical depth and $10 \%$ for the sky radiances are assumed.

\section{RESULTS}

We applied this approach to lidar measurements of MULIS and POLIS in Maisach around 2 UTC on 17 April 2010 in combination with AOD and almucantar measurements of a CIMEL photometer in Munich (about $25 \mathrm{~km}$ from the lidar) around 8:12 UTC on the same day. Two aerosol types were detected by the lidar, one type in the boundary layer up to about $1.7 \mathrm{~km}$ above ground and volcanic ash in a separate layer above. The intensive aerosol properties did not change 

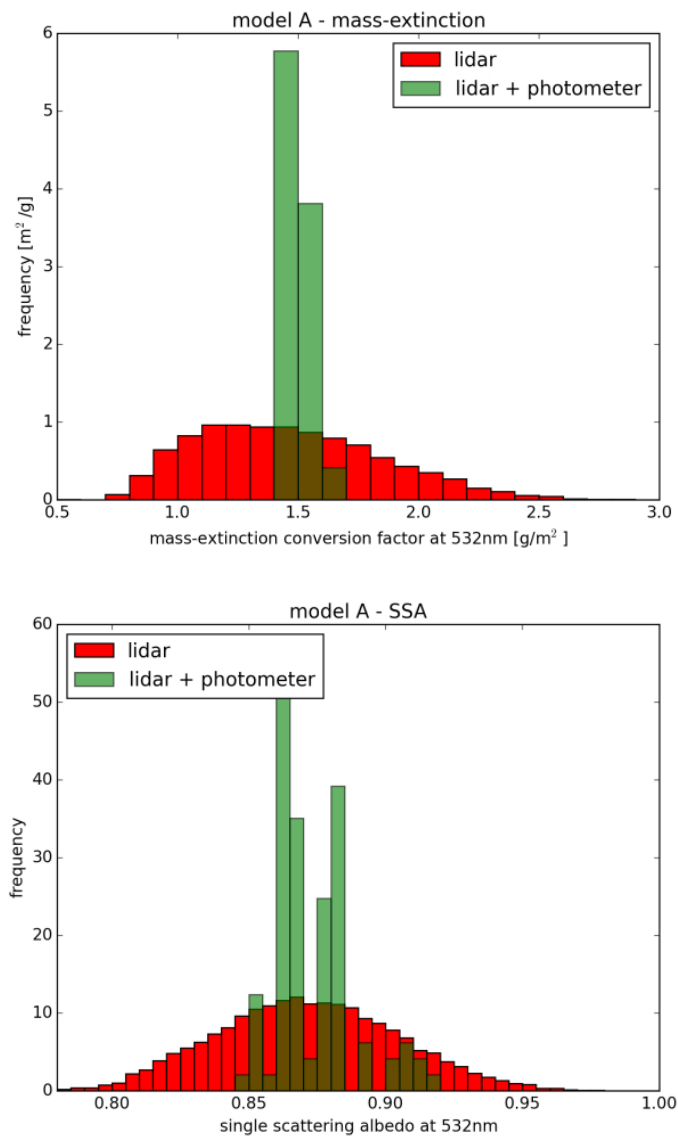

Fig. 2: Mass-extinction conversion factor (upper panel) and single scattering albedo (lower panel) of the solutions of the retrievals using model A.

during that time period, but the aerosol loading was variable.

As a consequence, the aerosol extinction coefficients during the photometer measurement are not known from lidar. To overcome this gap, measurements of a ceilometers located a few meters from the photometer are used to estimate the contributions of each layer. An optical depth of 0.30 at $\lambda=532 \mathrm{~nm}$ for the boundary layer aerosol and 0.25 for the volcanic ash aerosol provide good consistency with the ceilometer measurements in Munich. These layer optical depths were assumed subsequently, together with height-independent extinction coefficients within each layer. The boundary layer aerosol was characterized by low depolarization ratios, thus spherical particles were assumed for the retrieval of the boundary layer aerosol properties. 10000 lidar-compatible solution ensembles were retrieved for each layer (and each model).
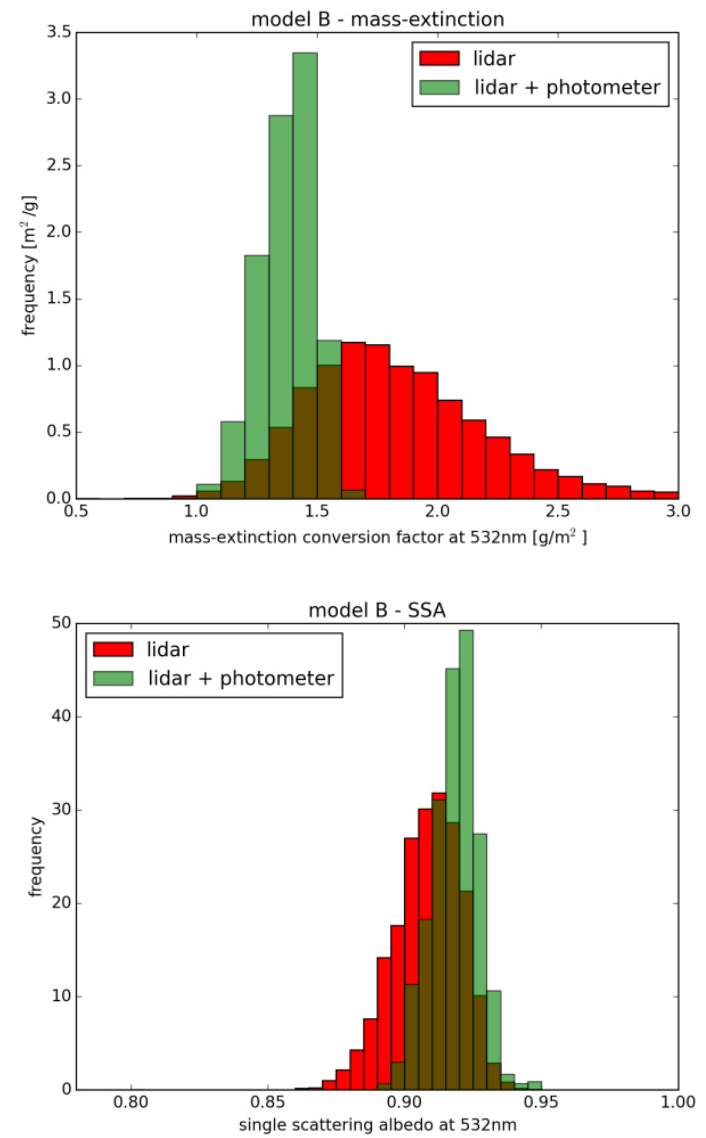

Fig. 3: Mass-extinction conversion factor (upper panel) and single scattering albedo (lower panel) of the solutions of the retrievals using model $B$.

Fig. 2 shows the frequency distributions for the retrieved solution ensembles for the ash layer when ash model A is used. The red bars illustrate the solutions of the lidar-only retrieval, whereas the green bars show the solutions of the combined lidar-photometer retrieval. The upper panel of Fig. 2 shows a significant reduction of the uncertainty of the mass-extinction conversion factor by about a factor 8 when the photometer measurements are considered (95\% uncertainty range shrinked from $0.87-2.28 \mathrm{~g} / \mathrm{m}^{2}$ to $\left.1.44-1.60 \mathrm{~g} / \mathrm{m}^{2}\right)$. The lower panel illustrates a reduction of the uncertainty of the single scattering albedo at $532 \mathrm{~nm}$ by about $50 \%$.

Fig. 3 is analogous to Fig. 2, however with the more complex ash model B used instead of model A. The width of the uncertainty range of the massextinction conversion factor is reduced by about $75 \%$ (from $1.21-2.82 \mathrm{~g} / \mathrm{m}^{2}$ to $1.15-1.56 \mathrm{~g} / \mathrm{m}^{2}$ ) if the additional photometer data is considered in 
case of model B. For the single scattering albedo a reduction of the uncertainties by $30 \%$ is found (from $0.882-0.929$ to $0.901-0.934$ ).

Comparison of the results using ash model A with results using model $\mathrm{B}$ reveals that the retrieved mass-extinction conversion factor is not strongly sensitive to the aerosol model employed. Only in the lidar-only case, a small shift towards larger mass-extinction conversion factors is found when the simpler model $\mathrm{A}$ is replaced by the complex and more realistic ash model $\mathrm{B}$. The uncertainties of the mass-extinction conversion factor are somewhat larger when using model B which can be understood in view of the increased number of model parameters of model B. For the single scattering albedo a significant shift towards larger values and a reduction of its uncertainties occurs if model A is replaced by model B.

The AOD data was found not to reduce the uncertainties in all investigated cases, thus the uncertainty reduction can be attributed mainly to the consideration of the sky radiance data. Only a very low fraction of the sampled atmospheric setups (about $10^{-5}$ ) were compatible with the AOD and sky radiance measurements in case of model A. The sampling frequency of solutions of the lidar-photometer retrieval was significantly higher (about $1.8 \cdot 10^{-2}$ ) in case of model B.

\section{CONCLUSIONS}

We investigated the benefit of photometer measurements and advanced aerosol models for a lidar-based aerosol retrieval. Our preliminary results show significantly reduced uncertainties for the retrieval of the mass-extinction conversion factor, and thus the aerosol mass concentration, when lidar measurements are complemented by photometer measurements. Improvements were found also for the single scattering albedo, but they were less significant than for the massextinction conversion factor.

Usage of the advanced model instead of the spheroid model leads to small changes of average retrieved parameters and the widths of their uncertainty ranges, indicating that the spheroid model is sufficient for volcanic ash retrievals. However, as the frequency of sampling retrieval solutions is much higher when the advanced model is used, a deeper look into the effect of the aerosol model is required for final conclusions.

\section{ACKNOWLEDGEMENT}

This work was funded by the LMUexcellent project EVAeNT.

\section{REFERENCES}

[1] Gasteiger, J., S. Groß, V. Freudenthaler, M. Wiegner, 2011: Volcanic ash from Iceland over Munich: mass concentration retrieved from ground-based remote sensing measurements, Atmos. Chem. Phys., 11, 2209-2223.

[2] Lopatin, A., O. Dubovik, A. Chaikovsky, P. Goloub, T. Lapyonok, D. Tanré, P. Litvinov, 2013: Enhancement of aerosol characterization using synergy of lidar and sun-photometer coincident observations: the GARRLiC algorithm, Atmos. Meas. Tech., 6, 2065-2088.

[3] Gasteiger, J., M. Wiegner, S. Groß, V. Freudenthaler, C. Toledano, M. Tesche, K. Kandler, 2011: Modeling lidar-relevant optical properties of complex mineral dust aerosols, Tellus B, 63, 725-741.

[4] Yurkin, M.A, A.G. Hoekstra, 2011: The discrete-dipole-approximation code ADDA: capabilities and known limitations, J. Quant. Spectrosc. Radiat. Transfer, 112, 2234-2247.

[5] Hess, M., P. Koepke, I. Schult, 1998: Optical Properties of Aerosols and clouds: The software package OPAC, Bull. Am. Met. Soc., 79, 831-844.

[6] Buras, R., T. Dowling, C. Emde, 2011: New secondary-scattering correction in DISORT with increased efficiency for forward scattering, $J$. Quant. Spectrosc. Radiat. Transfer, 112, 20282034.

[7] Mayer, B., A. Kylling, 2005: Technical note: The libRadtran software package for radiative transfer calculations - description and examples of use, Atmos. Chem. Phys., 5, 1855-1877. 